\title{
Increased fruit and vegetable consumption and immune function: the Ageing and Diet Intervention Trial (ADIT)
}

\author{
A. Gibson ${ }^{1}$, C. E. Neville ${ }^{1}$, S. E. C. M. Gilchrist, M. C. McKinley ${ }^{1}$, C. C. Patterson, J. D. Edgar ${ }^{2}$, \\ I. S. Young and J. V. Woodside ${ }^{1}$ \\ ${ }^{1}$ School of Medicine, Dentistry and Biomedical Sciences, Queen's University Belfast, Belfast BT12 6BJ, UK \\ and ${ }^{2}$ Regional Immunology Service, Belfast Health and Social Care Trust, Belfast BT12 6BN, UK
}

\begin{abstract}
The immune system undergoes a range of changes as individuals become elderly ${ }^{(1)}$. These changes may manifest as an increasing susceptibility to infection or a tendency to develop autoimmune or malignant disease ${ }^{(2)}$. Multiple underlying factors contribute to this phenomenon of immunological aging, and the present study has examined the possibility that inadequate diet may be one such contributing factor. Fruit and vegetable intake, which can be low in the elderly, is associated with reduced chronic disease risk. The study tested the hypothesis that increased fruit and vegetable intake may positively affect clinically-relevant measures of immune function.

Eighty-three healthy volunteers aged 65-85 years following a low-fruit and -vegetable diet (two or less portions per d) were recruited and randomised to continue following their normal diet or to consume at least five portions of fruit and vegetables daily for 16 weeks. At 12 weeks tetanus toxoid (0.5 ml intramuscularly (IM); Sanofi Pasteur MSD Ltd, Maidenhead, Berks., UK) and Pneumovax II (0.5 ml IM; Sanofi Pasteur MSD Ltd) were administered. Specific antibody binding to tetanus toxoid (total IgG) and pneumococcal capsular polysaccharide (total IgG) were assessed by ELISA at baseline, 12 weeks and 16 weeks. Biochemical markers of nutritional status (vitamin $\mathrm{C}$, lutein, zeaxanthin, $\beta$-cryptoxanthin, $\alpha$-carotene, $\beta$-carotene and lycopene) were assessed at baseline, 6 weeks, 12 weeks and 16 weeks to monitor compliance. Between group differences in change in endpoint were compared using independent samples t-tests.

Eighty-two participants completed the 16-week intervention. There was a significant difference in change in self-reported fruit and vegetable consumption between the groups consuming two portions per $\mathrm{d}$ and five portions per $\mathrm{d}$ (mean change in number of portions; two portions per d, 0.41 (SD 0.79); five portions per d, 4.61 (SD 1.38); $P<0.001$ ), and this finding was reflected in significantly larger increases in vitamin $\mathrm{C}(P<0.001)$, lutein $(P<0.05)$ and $\beta$-cryptoxanthin $(P<0.01)$ status in those consuming five portions per $\mathrm{d}$ compared to those consuming two portions per $\mathrm{d}$. There was no difference in antibody binding to tetanus toxoid between the two intervention groups (data not shown), but antibody binding to pneumococcal capsular polysaccharide increased more in the group consuming five portions per d than in the group consuming two portions per d (geometric mean change from baseline, which represents percentage of baseline; two portions per d, 200 (95\% CI -352, 429); five portions per d, 315 (95\% CI 1, 996); $P=0.036)$.

In the present study there is a greater specific antibody response to Pneumovax II vaccination in those consuming five portions of fruit and vegetables per d compared with those consuming two portions of fruit and vegetables per $\mathrm{d}$. Thus increased fruit and vegetable intake may improve antibody response to vaccination in older individuals.
\end{abstract}

1. Meydani M (2002) Proc Nut Soc 61, 165-171.

2. Dorshkind K, Montecino-Rodriguez E \& Signer RAJ (2009) Nat Rev Immunol 9, 57-62. 